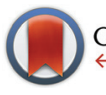

CrossMark

\& click for updates

Cite this: Polym. Chem., 2016, 7, 5414

Received 1st June 2016 Accepted 4th August 2016

DOI: $10.1039 / c 6 p y 00937 a$

www.rsc.org/polymers

\section{Two in one: use of azide functionality for controlled photo-crosslinking and click-modification of polymer microspheres $\dagger$}

\author{
Marco Albuszis, ${ }^{a}$ Peter J. Roth, ${ }^{{ }^{b}}$ Werner Pauer ${ }^{\star a}$ and Hans-Ulrich Moritz ${ }^{\star^{a}}$
}

Spherical, micrometer-sized, azide-functional particles were produced through dispersion copolymerization of styrene and vinylbenzyl azide (VBA, 1-100 wt\% of monomer feed) in ethanol in the presence of stabilizers. The obtained microspheres were characterized by SEM, disc centrifuge, FT-IR and NMR spectroscopy, elemental analysis, DSC, and TGA, had measured azide loadings of up to $5.58 \mathrm{mmol} \mathrm{g}^{-1}$, and average diameters that decreased with increasing azide content from 2.8 to $0.8 \mu \mathrm{m}$. Microspheres were irradiated at a wavelength of $254 \mathrm{~nm}$ resulting in crosslinking based on azide-to-nitrene decomposition and subsequent $\mathrm{C}-\mathrm{H}$ insertion and $\mathrm{C}=\mathrm{C}$ addition reactions. The conversion of azide functionality was monitored by FT-IR spectroscopy, elemental analysis, and DSC and was found to roughly follow firstorder kinetics with increased rates found for microspheres with lower azide contents. Photo-crosslinking preserved shapes and size distributions and, above a crosslinking degree of $10 \%$, prevented microsphere dissolution in good solvents. By controlling the irradiation time, the amount of azide consumed for photo-crosslinking could be precisely adjusted. Residual azide groups spared during the irradiation were shown to be amenable to highly efficient CuAAC click modification with a fluorescent dye, Rhodamine B propargyl ester. Given the demand for functional crosslinked microspheres and the inherent difficulties associated with common synthetic strategies in producing such materials, this methodology based on two orthogonal chemistries of the azide functionality provides simple access to well-defined microspheres with customizable degrees of crosslinking and functional group densities.

\section{Introduction}

Spherical, polymer-based, micrometer-sized particles (microspheres) have many applications in materials science and technology including affinity bioseparation, ${ }^{1}$ liquid chromatography support materials, ${ }^{2,3}$ drug and enzyme carriers for medical applications, ${ }^{4}$ model systems to study rheology and crystallization of colloidal materials, ${ }^{5-7}$ and absorbents. ${ }^{8}$ Apart from size, type of polymer, and porosity, additional parameters that are crucial for applicability are crosslinking (which improves mechanical stability and prevents disintegration in solvents for the base polymer $)^{6,9-11}$ and chemical surface functionality (which improves compatibility with solvents/host

\footnotetext{
${ }^{a}$ Institute for Technical and Macromolecular Chemistry, University of Hamburg, Bundesstraße 45, 20146 Hamburg, Germany.E-mail: pauer@chemie.uni-hamburg.de, moritzhu@chemie.uni-hamburg.de

${ }^{b}$ Department of Chemistry, Faculty of Engineering and Physical Sciences, University of Surrey, Guildford, Surrey, GU2 7XH, UK. E-mail: p.roth@surrey.ac.uk

$\dagger$ Electronic supplementary information (ESI) available. See DOI: 10.1039/ c6py00937a
}

materials and is essential for immobilizing labels or payloads). ${ }^{12}$

A range of protocols for microsphere synthesis is available and includes the classical dispersion, emulsion, and suspension techniques, ${ }^{13-15}$ polymerization-induced self-assembly (PISA), ${ }^{16-18}$ as well as multistep seed-swelling procedures. ${ }^{19-22}$ Most of these methods, however, are not well suited for the direct synthesis of crosslinked and functionalized microspheres. Dispersion polymerization, during which a homogeneous solution of monomer, initiator, and stabilizers produces insoluble microspheres, is a well-established and simple method for the preparation of micrometer-sized, nonporous, monodisperse particles. ${ }^{23-29}$ However, this method has limitations in the preparation of crosslinked particles, with the presence of crosslinkers during the nucleation step typically resulting in non-spherical or coagulated particles while step-wise addition of crosslinkers after nucleation allows only for relatively low crosslinking densities. ${ }^{11,30-34}$ Other methods, seed-swelling suspension polymerizations, for example, offer excellent control over the crosslinking density (formulations with $100 \%$ crosslinkers are possible), ${ }^{19-22}$ but the incorporation of functional or reactive comonomers can 
result in the formation of irregular, hollow, or collapsed particles. ${ }^{35}$ Crosslinking and/or chemical modification of microspheres are thus often performed in additional postpolymerization modification steps. ${ }^{12,26,36-42}$

An expedient functional group for such post-modification is the azide functionality. Azide-functional particles (whether produced through dispersion polymerization or other methods) are gaining increased attention ${ }^{35,43-46}$ due to the versatility and scope of the Cu-catalyzed alkyne-azide cycloaddition (CuAAC) click reaction. ${ }^{47}$ It is worth noting that the azide functionality is usually installed into pre-made particles (typically through substitution with sodium azide on chloromethyl groups) and that this reaction often suffers from poor conversions, due, in part, to solubility limitations. Only very few published reports have used a direct copolymerization of azide-functional comonomers (such as 4-vinylbenzyl azide) for the preparation of azide-functional microspheres and, to the best of our knowledge, dispersion polymerization based on 4-vinylbenzyl azide has not yet been described. ${ }^{35,46,48}$

The azide functionality also allows for post-synthesis photocrosslinking, ${ }^{42,49}$ which has been demonstrated on soluble polymers $^{50}$ and polymer films. ${ }^{51,52}$ This reaction is based on the decomposition of the azide moiety upon UV-irradiation, loss of a nitrogen molecule, and formation of a nitrene intermediate. Being highly reactive, the nitrene species can undergo several reactions with the surrounding material (often a combination of reaction pathways is found) predominantly through $\mathrm{C}-\mathrm{H}$ insertion and $\mathrm{C}=\mathrm{C}$ addition reactions, which result in crosslinks. To the best of our knowledge, the azide photosensitivity has not yet been used for the crosslinking of polymer microspheres prepared through heterogeneous polymerization methods.

The purpose of the present study is twofold. Firstly, successful dispersion copolymerization of 4-vinylbenzyl azide (VBA) with styrene in ratios ranging from 1-100 wt\% VBA is demonstrated to produce microspheres with a tunable azide content. Compared to our previous work in which azide functionality was installed into microspheres through postpolymerization modification of residual vinyl groups of divinylbenzene-based particles $^{43}$ or through polymerization of VBA inside PS template spheres, ${ }^{35}$ the current method leads to much higher measured azide loadings of up to $5.58 \mathrm{mmol} \mathrm{g}^{-1}$. It is further shown that this direct copolymerization is favorable over the post-synthesis installation of azide into 4-vinylbenzyl chloride comonomers, because the chloromethyl groups were found to be susceptible to unwanted nucleophilic substitution by the solvent ethanol resulting in a loss of reactive groups during the polymerization step. Secondly, photo-crosslinking of azidefunctional microspheres is demonstrated to yield well-defined particles that can be dispersed without disintegration in good solvents. Importantly, we found that the amount of azide groups consumed for photo-crosslinking can be precisely tuned through controlling the irradiation time. As a result, the crosslinking density and the amount of residual azide groups can be controlled. This residual azide functionality is demonstrated to be amenable to chemical modification through a near-quantitative CuAAC click modification with an alkynefunctional fluorescent dye, Rhodamine B. This study emphasizes how the azide functionality, as a single functional group, can be installed, without loss, into well-defined microspheres and can be exploited for both a controllable degree of crosslinking and for surface modification, providing simple access to well-defined functional materials expected to facilitate many of the aforementioned applications.

\section{Experimental section}

\section{Materials}

Unless otherwise noted, chemicals were purchased from Sigma Aldrich, Merck Chemicals, or Acros Organics in analytical grade and were used without further purification. Styrene (S) was distilled in vacuum to remove inhibitors and impurities directly before use. 2,2'-Azobis-(2-isobutyronitrile) (AIBN) was recrystallized from methanol. 4-Vinylbenzyl chloride was of technical grade $(90 \%)$ and distilled in vacuum directly before use. Rhodamine B propargyl ester and 4-vinylbenzyl azide (VBA) were prepared as published elsewhere. ${ }^{35}$

\section{Dispersion polymerization synthesis of poly(styrene-co-4- vinylbenzyl azide), P(S-co-VBA) microspheres}

Reactive, azide containing microspheres were produced following a typical literature procedure. ${ }^{43}$ Briefly, reactions were performed in 3-necked $250 \mathrm{ml}$ double walled glass reactors connected to a thermostat and placed on a shaking plate without additional stirrers. A typical polymerization procedure is given as follows: solvent ethanol (135 g) and stabilizers polyvinylpyrrolidone (PVP-K30, $M_{\mathrm{n}}=40 \mathrm{~kg} \mathrm{~mol}^{-1}, 1.5 \mathrm{~g}$ ) and Aliquat 336 ( $0.6 \mathrm{~g}$ ) were mixed in a flask overnight and transferred into a reactor followed by purging with argon for 30 minutes while shaking at $150 \mathrm{rpm}$. The temperature was increased to $70{ }^{\circ} \mathrm{C}$. A monomer mixture (15 g total) consisting of AIBN (0.3 g), styrene, and 4-vinylbenzyl azide (in variable mass ratios) was purged with argon for 15 minutes in a separate flask and transferred into the preheated reactor. The polymerization was run for $24 \mathrm{~h}$ with shaking at $150 \mathrm{rpm}$. The mixture was cooled to room temperature and the microspheres were purified by repeated washing-centrifugation steps using ethanol, hot water, and ethanol again for redispersing before drying in vacuum. ${ }^{1} \mathrm{H} \mathrm{NMR}\left(\mathrm{CDCl}_{3}\right) \delta / \mathrm{ppm}=7.00,6.50(\mathrm{bm}$, $\mathrm{Ph}) ; 4.15\left(-\mathrm{CH}_{2} \mathrm{~N}_{3}\right) ; 2.28-0.72$ (bm, backbone). Molar copolymer compositions were determined by comparison of the integrals of the benzylic protons $(2 \mathrm{H})$ of VBA to those of the phenylic side chains ( $5 \mathrm{H}$ for $\mathrm{S}, 4 \mathrm{H}$ for VBA) and the backbone (3 H). Results from FT-IR, SEM, DSC, TGA, and elemental analysis are presented below.

Poly(styrene-co-4-vinylbenzyl chloride) microspheres were prepared in analogy by substituting VBA for 4-vinylbenzyl chloride. 


\section{Microsphere photo-crosslinking}

Azide-functional microspheres ( $0.2 \mathrm{~g})$ were suspended in water (200 $\mathrm{mL}$ ) using sonication and stirred mechanically in a narrow glass cylinder. A Heraeus UV-2 laboratory reactor system consisting of a cooling tube and dip tube made of quartz equipped with a TQ 150 immersion emitter (output 150 $\mathrm{W}$, emission maxima at 254, 313, and $366 \mathrm{~nm}$ ) was dipped directly into the stirred dispersion. Irradiation was done at room temperature for a predetermined amount of time before particles were isolated by centrifugation and washing.

\section{Click modification of partially photo-crosslinked P(S-co-VBA) microspheres}

Partially photo-crosslinked microspheres containing residual azide functionality (100 $\mathrm{mg}$ ) were dispersed in a mixture of DMF ( $5 \mathrm{~mL}$ ), $N, N, N^{\prime}, N^{\prime}$-tetramethylenethylendiamine (TMEDA), $\mathrm{CuBr}$, and triethylamine (approx. 1 eq. each with regards to azide functionality) and the mixture was purged with argon for 5 min. Rhodamine B propargyl ester (3 eq. with regards to azide functionality) was added, the reaction flask sealed and stirred for $24 \mathrm{~h}$ at $50{ }^{\circ} \mathrm{C}$. Modified microspheres were isolated by filtering and washing with DMF, water, ethanol, and acetone (25 $\mathrm{mL}$ each) until washings remained colorless, followed by drying in vacuum. Analytical data is presented and discussed in the main text.

\section{Characterization}

Surface morphology and uniformity of particles were determined by scanning electron microscopy (SEM, LEO1525). Particles were dispersed in ethanol with sonication and spread onto an aluminum specimen stub. After drying, samples were coated with a thin layer of carbon in vacuum. Number-average particle diameters $\left(d_{\mathrm{n}}\right)$ and diameter dispersities $\left(\bigoplus_{\mathrm{d}}\right)$ were calculated by measuring at least 100 particles using image J software. The coefficient of variation (CV), weight- $\left(d_{\mathrm{w}}\right)$ and number-average $\left(d_{\mathrm{n}}\right)$ diameters were calculated using the following equations where $n_{i}$ is the number of particles of diameter $d_{i}$ and $N$ is the total number of particles. ${ }^{36,53}$

$$
\begin{gathered}
d_{\mathrm{w}}=\frac{\sum n_{i} d_{i}{ }^{4}}{\sum n_{i} d_{i}{ }^{3}} \\
d_{\mathrm{n}}=\frac{\sum n_{i} d_{i}}{\sum n_{i}} \\
\bigoplus_{\mathrm{d}}=\frac{d_{\mathrm{w}}}{d_{\mathrm{n}}} \\
\mathrm{CV}=\frac{\left[\left(\sum_{i=1}^{N}\left(d_{i}-d_{\mathrm{n}}\right)^{2}\right) /(N-1)\right]^{1 / 2}}{d_{\mathrm{n}}} \times 100
\end{gathered}
$$

Chemical transformations of microspheres were monitored by FT-IR spectroscopy on an ATR-FT-IR spectrometer Nicolet iS10 (Thermo Fisher Scientific). Spectra were baseline corrected for easier interpretation.
Solution NMR spectra were measured on a Bruker Avance 400 spectrometer using $\mathrm{CDCl}_{3}$ as solvent and the internal solvent signal $\delta\left(\mathrm{CHCl}_{3}\right)=7.26 \mathrm{ppm}$ as reference. Solid state NMR experiments were performed on a Bruker Avance 400 spectrometer, equipped with a $4 \mathrm{~mm}$ double resonance probe. ${ }^{13} \mathrm{C}$ cross polarization (CP) magic angle spinning (MAS) spectra were acquired using ramped polarization transfer at an operating frequency of $100.66 \mathrm{MHz}$. The experimental conditions were: ${ }^{1} \mathrm{H} 90^{\circ}$ pulse length $4.0 \mu \mathrm{s}$, contact time $2 \mathrm{~ms}$, repetition delay $5 \mathrm{~s}$, and spinning rate $14 \mathrm{kHz}$. Two-phase modulation (TPPM) decoupling was used during the acquisition. All the measurements were carried out at room temperature.

CHN elemental analysis was performed on EuroVector Euro EA and Elementar Vario EL III instruments. Combustion gases were detected by a gas chromatography (Euro EA) or a head conductivity detector (Vario EL III).

Differential scanning calorimetry (DSC), performed on a Mettler Toledo DSC 1 Star System, was used to determine glass transition temperatures $\left(T_{\mathrm{g}}\right)$ and the thermal behavior of the polymer samples. The heating rate was $10{ }^{\circ} \mathrm{C} \mathrm{min}^{-1}$ and experiments were done under nitrogen atmosphere with a flow

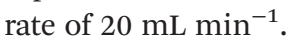

Thermogravimetric analysis (TGA) of samples was performed on a Mettler Toledo TGA 1 Star System thermogravimeter with a heating rate of $10{ }^{\circ} \mathrm{C} \mathrm{min}^{-1}$ in a nitrogen atmosphere with a flow rate of $20 \mathrm{~mL} \mathrm{~min}^{-1}$.

\section{Results and discussion}

This study comprised the synthesis of azide functional particles, their partial photo-crosslinking, and the click-modification of residual azide functionality, Scheme 1.

\section{Microsphere synthesis}

Styrene-based microspheres were prepared by dispersion polymerization using ethanol as solvent, AIBN as initiator, and PVP-K30 and Aliquat 336 as stabilizers in fixed mass ratios relative to monomers. ${ }^{35}$ As mentioned above, arguably the most common method to introduce azide functionality into microspheres is the postpolymerization reaction of vinylbenzyl chloride groups with sodium azide. Following this protocol, a

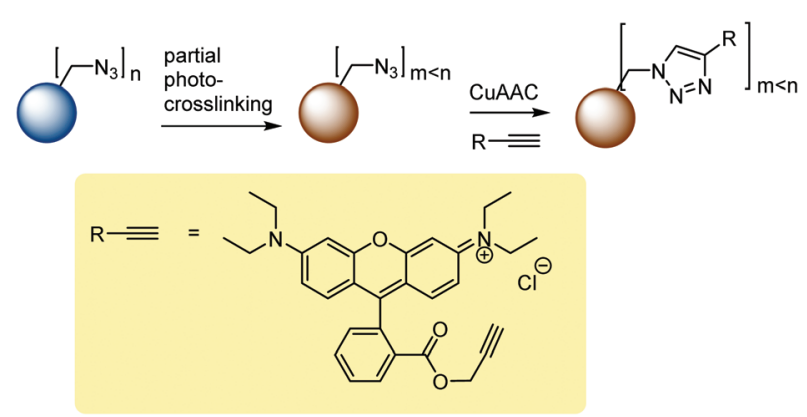

Scheme 1 Reaction scheme. 
series of chloride-functional microspheres was prepared through dispersion copolymerization of styrene (S) and 4-vinylbenzyl chloride (VBC) with $\mathrm{S}$ : VBC mass ratios ranging from $99: 1$ to $0: 100$. Uniform microspheres of $2-3 \mu \mathrm{m}$ diameter were obtained as evidenced by SEM imaging (Fig. S1 in the ESI $\dagger$ ). However, ${ }^{1} \mathrm{H}$ NMR analysis run on $\mathrm{CDCl}_{3}$ solutions of the copolymers, Fig. S2, $\dagger$ suggested that up to $16 \%$ of chloromethyl functionality was lost to nucleophilic substitution with the solvent ethanol, as judged by the appearance of a broad signal at $\delta=3.51 \mathrm{ppm}$ attributed to the benzylic ethyl ether ( $\mathrm{Ar}-\mathrm{CH}_{2}-\mathrm{OR}$ ) (see Scheme 2) and as confirmed through 2D NMR analysis (not shown).

The observed percentage of chloride-ethanol substitution of $12-16 \mathrm{~mol} \%$ did not depend strongly on the VBC feed content but was reduced to $9 \mathrm{~mol} \%$ in a trial experiment run without Aliquat 336 (a quaternary ammonium bromide species) as stabilizer, suggesting that the bromide anion may facilitate the substitution reaction through formation of a bromide intermediate, Fig. S3. $\dagger$

These results encouraged us to investigate the direct copolymerization of 4-vinylbenzyl azide (VBA) and styrene under the same experimental conditions (including the use of Aliquat 336 as stabilizer). Ten microsphere samples with S:VBA comonomer feed ratios between 99:1 and 0:100 wt\% were prepared and characterized visually and by ${ }^{1} \mathrm{H}$ solution NMR spectroscopy, scanning electron microscopy (SEM), CHN elemental analysis (EA), FT-IR spectroscopy, thermogravimetric analysis (TGA) and differential scanning calorimetry (DSC), see Scheme 2B and Table 1.

Dried microspheres presented as powders that were colorless (low VBA content) to yellow (higher VBA content), see Fig. S4.† $\dagger$

${ }^{1} \mathrm{H}$ NMR spectroscopic analysis of microspheres dissolved in $\mathrm{CDCl}_{3}$, Fig. 1, showed the expected signals of the aromatic region $(\delta=7.20-6.40 \mathrm{ppm})$ and backbone (1.90-1.10 ppm), and indicated the successful incorporation of VBA units through the appearance of a signal at $4.22 \mathrm{ppm}$, attributed to the $\mathrm{Ar}-\mathrm{CH}_{2} \mathrm{~N}_{3}$ resonance. Notably, ${ }^{1} \mathrm{H}$ NMR spectra gave no evidence of side reactions with the solvent and the integration of the
A<smiles>C=Cc1ccccc1</smiles><smiles>C=Cc1ccc(CCl)cc1</smiles>

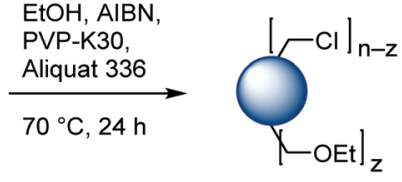

B

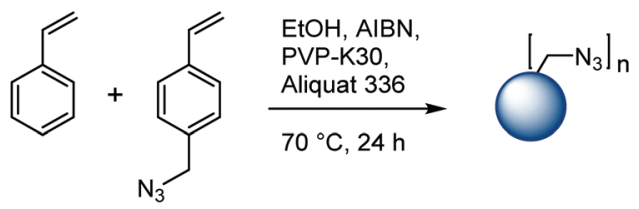

Scheme 2 (A) Side reaction through ethanolysis during the synthesis of chloromethyl-functional microspheres (which can serve as precursors for azide-functional microspheres) and (B) microsphere synthesis through dispersion polymerization of VBA and styrene.
Table 1 Overview of microspheres samples

\begin{tabular}{|c|c|c|c|c|c|c|}
\hline \multirow[b]{2}{*}{ Code } & \multicolumn{2}{|l|}{ Feed } & \multicolumn{3}{|c|}{ SEM analysis } & \multirow{2}{*}{$\begin{array}{l}\text { CHN analysis } \\
\text { Azide }^{d} \\
{\left[\mathrm{mmol} \mathrm{g}^{-1}\right]}\end{array}$} \\
\hline & $\begin{array}{l}\text { Styrene } \\
{[\mathrm{wt} \%]}\end{array}$ & $\begin{array}{l}\text { VBA } \\
{[w t \%]}\end{array}$ & $\begin{array}{l}d_{\mathrm{n}}{ }^{a} \\
{[\mu \mathrm{m}]}\end{array}$ & $D_{\mathrm{d}}^{b}$ & $\begin{array}{l}\mathrm{CV}^{c} \\
{[\%]}\end{array}$ & \\
\hline $\mathrm{MS}_{1}$ & 99 & 1 & 2.77 & 1.002 & & 0.12 \\
\hline $\mathrm{MS}_{2}$ & 98 & 2 & 2.63 & 1.005 & 7.3 & 0.17 \\
\hline $\mathrm{MS}_{5}$ & 95 & 5 & 2.59 & 1.004 & 6.4 & 0.36 \\
\hline $\mathrm{MS}_{10}$ & 90 & 10 & 2.49 & 1.002 & 4.6 & 0.66 \\
\hline $\mathrm{MS}_{20}$ & 80 & 20 & 2.12 & 1.07 & 23.1 & 1.31 \\
\hline $\mathrm{MS}_{30}$ & 70 & 30 & 1.68 & 1.28 & 52.8 & 1.95 \\
\hline $\mathrm{MS}_{40}$ & 60 & 40 & 1.96 & 1.16 & 40.45 & 2.55 \\
\hline $\mathrm{MS}_{50}$ & 50 & 50 & 1.34 & 1.06 & 25.9 & 3.19 \\
\hline $\mathrm{MS}_{75}$ & 25 & 75 & 1.28 & 1.08 & 29.07 & 4.26 \\
\hline $\mathrm{MS}_{100}$ & 0 & 100 & 0.84 & 1.08 & 29.6 & 5.58 \\
\hline
\end{tabular}

${ }^{a}$ Number-average particle diameter. ${ }^{b}$ Dispersity of the particle diameter. ${ }^{c}$ Coefficient of variation of the particle diameter. ${ }^{d}$ Molar azide content calculated from elemental nitrogen analysis.

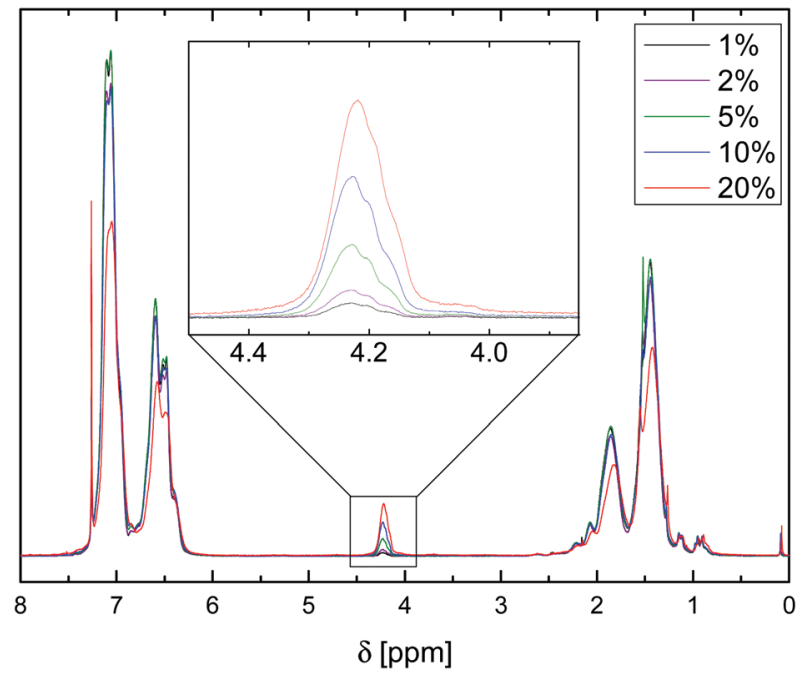

Fig. $1{ }^{1} \mathrm{H}$ NMR spectra of dissolved $\mathrm{P}(\mathrm{S}-\mathrm{co}-\mathrm{VBA})$ microspheres with the signals associated with the azidomethyl groups enlarged for microsphere samples with varying VBA feed contents.

benzyl groups and comparison with the aromatic and backbone signals agreed well with the $\mathrm{S}$ : VBA comonomer feed ratios.

SEM analysis, Fig. 2 and 3, showed spherical particles for all S-VBA compositions and typical diameters of approx. $2.7 \mu \mathrm{m}$. For VBA contents below $20 \mathrm{wt} \%$, microspheres were highly regular with monomodal size distributions and size dispersities as low as 1.002 (Table 1). For VBA contents above $20 \mathrm{wt} \%$, samples showed bimodal size distributions featuring large particles of approx. $2.8 \mu \mathrm{m}$ diameter and a population of smaller spheres with diameters around $1 \mu \mathrm{m}$, see Fig. 4 for two representative size histograms. At very high VBA feed ratio, the average microsphere diameter dropped to $0.8 \mu \mathrm{m}$ with the larger particles in sample $\mathrm{MS}_{100}$ measuring approx. $1.2 \mu \mathrm{m}$ in diameter. A small degree of particle agglomeration was observed for samples of highest VBA content. Several factors have been shown to influence particle sizes and distributions 

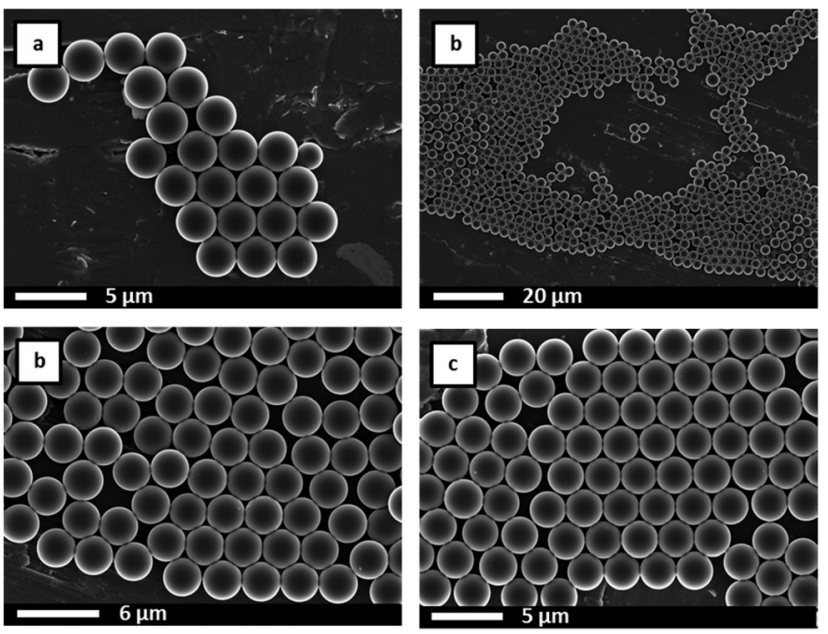

Fig. 2 SEM images of microsphere samples (a) $\mathrm{MS}_{1}$; (b) $\mathrm{MS}_{5}$ (two images); and (c) $\mathrm{MS}_{10}$

and include stabiliser molecular weight, stabiliser concentration, and solvent polarity. ${ }^{54}$ In the present case, the observed sizes and size distributions are likely caused by the polar nature of VBA (compared to S) and a presumed incompatibility of the growing S-co-VBA oligomers with the ethanol-S-VBA solvent mixture which would lead to earlier precipitation and a larger number of smaller particles. ${ }^{27,28,54}$

Disc centrifuge measurements, Fig. S5, $\dagger$ expectedly indicated narrow particle size distributions for samples with low to medium VBA contents. For higher VBA contents, however, results were inconclusive, presumably due to a slight crosslinking of polymers through thermal azide degradation and nitrene-based coupling reactions during polymerization..$^{55}$ Indeed, solubility tests of samples with more than $50 \mathrm{wt} \%$ VBA in THF (a good solvent for the base copolymers) resulted in the swelling (rather than dissolution) of particles suggesting crosslinking. With 20-50 wt\% VBA, microsphere samples dissolved partially with insoluble components forming a swollen gel. With less than $20 \mathrm{wt} \%$ of VBA, on the other hand, microspheres dissolved fully in THF and $\mathrm{CDCl}_{3}$. In order to estimate the amount of azide loss and degree of crosslinking formed during the polymerization with higher certainty than through ${ }^{1} \mathrm{H}$ NMR analysis, elemental analysis was performed of all microsphere samples. Taking into account the molar masses of monomers (and the amount of nitrogen contributed by the
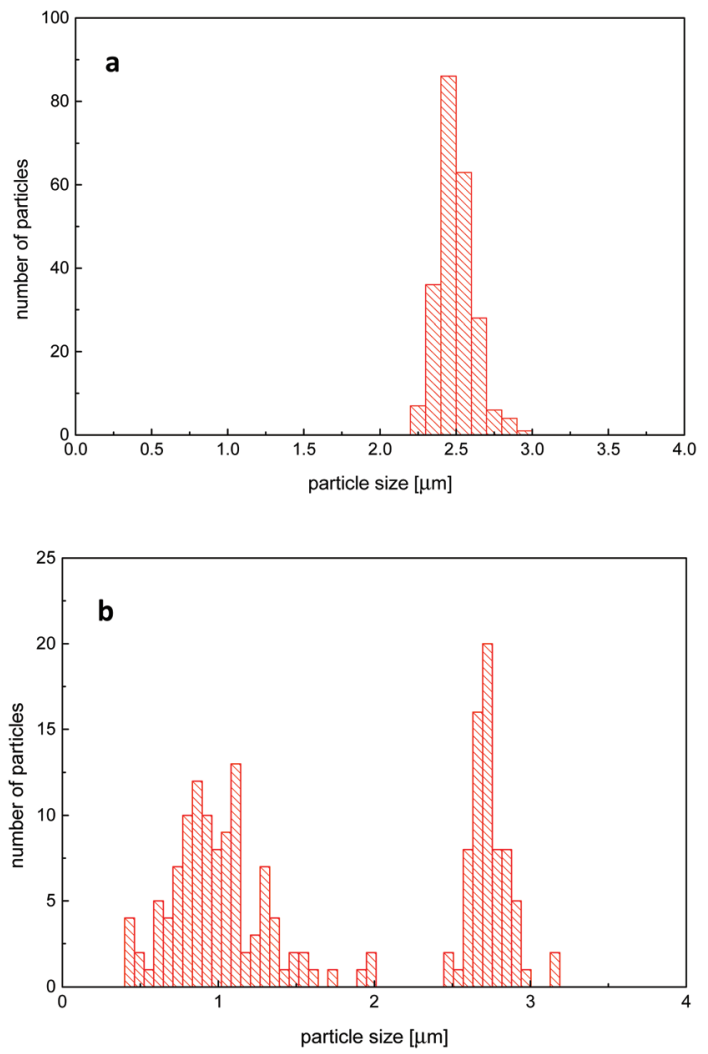

Fig. 4 Size distribution histograms of (a) $\mathrm{MS}_{10}$ and (b) $\mathrm{MS}_{30}$.

cyanopropyl initiating group originating from AIBN), the measured nitrogen content was used to calculate microsphere azide loadings (Table 1) and the ratio of found-to-expected azide content. These values, plotted in Fig. 5, ranged between 0.93 and 1.00 corroborated an, albeit small, loss of azide functionality during polymerization for samples of higher VBA content, in agreement with previous studies on thermal azide degradation in soluble polymer samples. ${ }^{55}$ With regards to the synthesis of azide-functional microspheres, we recently described the preparation of porous microspheres through a templated copolymerization of S, VBA, and divinylbenzene inside PS seed particles, which lead to found-to-expected azide content ratios as low as 0.37 and below 0.70 for most samples. In spite of some loss (and associated slight crosslinking) observed in the present study, dispersion (co)polymerization of VBA presents a viable avenue toward microspheres with
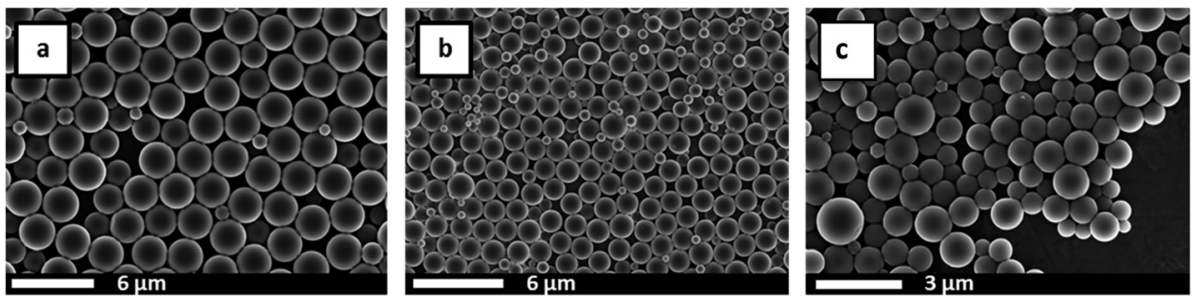

Fig. 3 SEM images of microsphere samples (a) $M S_{20}$; (b) $M S_{40}$ and (c) $M S_{100}$. 


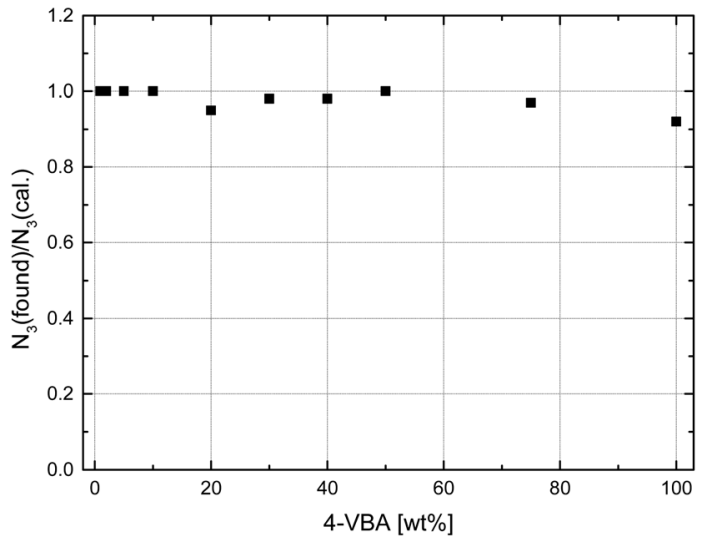

Fig. 5 Ratio of azide content found by elemental analysis of microspheres to expected azide content calculated from the feed ratio in dependence of VBA feed.

measured azide loadings as high as $5.58 \mathrm{mmol} \mathrm{g}^{-1}$ (see Table 1).

As eventual photo-crosslinking of particles was intended, a low degree of crosslinking for the VBA-rich samples was not an impediment. It is, however, for sake of synthetic scope, briefly mentioned that we additionally performed dispersion polymerization syntheses of azide-rich particles (up to $100 \%$ VBA) at $30{ }^{\circ} \mathrm{C}$ using $2,2^{\prime}$-azobis(4-methoxy-2,4-dimethyl valeronitrile) (V-70) as low-temperature radical initiator under otherwise unchanged reaction conditions. ${ }^{56}$ All of these microspheres dissolved in THF and chloroform, indicating the successful synthesis of microspheres with very high azide loadings without any observed crosslinking.

FT-IR analysis of microspheres, Fig. S6, $\uparrow$ showed the expected spectra of the $\mathrm{P}(\mathrm{S}-c o$-VBA $)$ base material including a strong band at $2095 \mathrm{~cm}^{-1}$ associated with the $\mathrm{N}=\mathrm{N}=\mathrm{N}$ asymmetric stretching and characteristic bands at $\nu / \mathrm{cm}^{-1}=1246$, $1050,878,816$ and 755 that varied with the comonomer feed ratio. The azide band served as an expedient means for the quantification of the azide content (see below).

Given this first dispersion-based synthesis of microspheres with very high azide contents, their potential in the materials science field, and the known thermal lability of the azide functionality, the thermal properties of all samples were characterized by DSC and TGA. DSC was used to determine glass transition temperatures, $T_{\mathrm{g}}$, and the energy, $E$, released as exothermic heat flow during the thermal decomposition of the azide groups of all samples. A representative DSC curve, measured of sample $\mathrm{MS}_{50}$, is given in Fig. 6 (bottom curve) and values of $T_{\mathrm{g}}$ and $E$ are plotted vs. VBA feed ratio in Fig. 7 . The glass transition temperature showed a linear dependence on the S-VBA composition, decreasing from $102{ }^{\circ} \mathrm{C}$ found for sample $\mathrm{MS}_{1}$ (close to the literature value of $100{ }^{\circ} \mathrm{C}$ for homo$\mathrm{PS})^{57}$ to $66^{\circ} \mathrm{C}$ measured for sample $\mathrm{MS}_{100}$, presumably due to increased sterical hindrance associated with the azidomethyl groups and less compact packing of polymer chains. The measured heat flow during azide decomposition varied in a

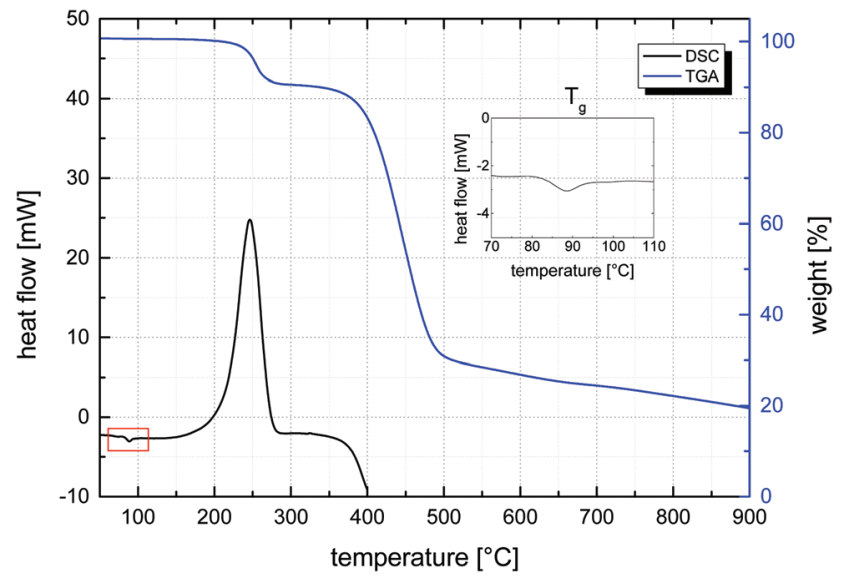

Fig. 6 Representative DSC (bottom curve, left axis) and TGA (top curve, right axis) data of $\mathrm{MS}_{50}$ showing the glass transition temperature (DSC, inset), the exothermic azide decomposition around $250^{\circ} \mathrm{C}$, the polymer decomposition at $380-480{ }^{\circ} \mathrm{C}$, and a residue of approx. $20 \mathrm{wt} \%$ at $900{ }^{\circ} \mathrm{C}(\mathrm{TGA})$.

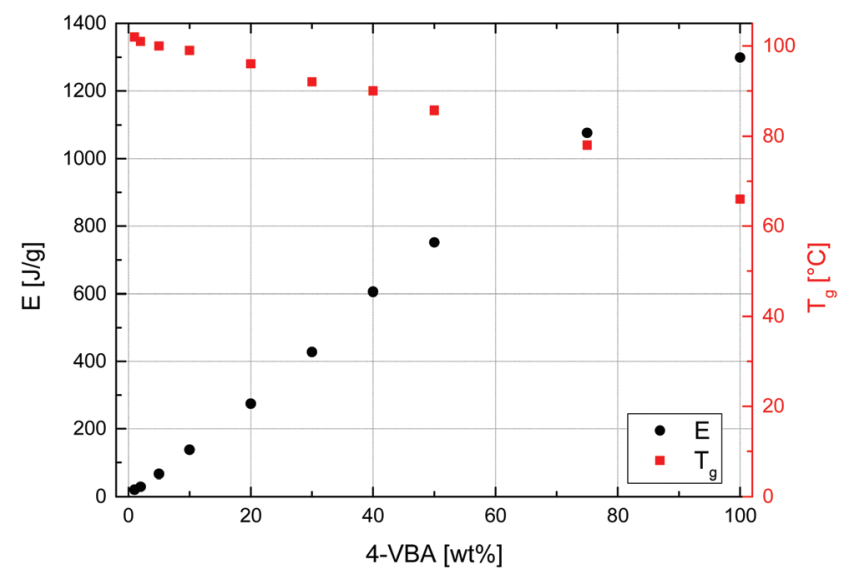

Fig. 7 Glass transition temperatures ( $T_{\mathrm{g}}$, squares, right axis) and exothermic heat flow ( $E$, circles, left axis) during azide decomposition measured by differential scanning calorimetry vs. VBA feed.

linear fashion with azide content, increasing from $20 \mathrm{~J} \mathrm{~g}^{-1}$ for sample $\mathrm{MS}_{1}$ to $1299 \mathrm{~J} \mathrm{~g}^{-1}$ for sample $\mathrm{MS}_{100}$. Importantly, this data was used as a calibration and allowed for the estimation of azide contents in microspheres after partial crosslinking (see below). Above $350{ }^{\circ} \mathrm{C}$, the onset of an endothermic peak indicated the decomposition of the polymer.

A representative decomposition curve obtained by TGA is shown in Fig. 6 (top curve) and curves of all samples are shown in Fig. S7.† In agreement with DSC, the loss of the azide group was observed between $200-280{ }^{\circ} \mathrm{C}$ through a mass loss corresponding to approximately two thirds of the nitrogen content, in agreement with the loss of one $\mathrm{N}_{2}$ molecule per $-\mathrm{N}_{3}$ group. With higher azide contents, the temperature of highest azide decomposition rate decreased from around $270{ }^{\circ} \mathrm{C}$ to $240{ }^{\circ} \mathrm{C}$ (see ESI $\dagger$ ), making high-concentration azide microspheres somewhat more susceptible to thermal 
crosslinking at temperatures above $200{ }^{\circ} \mathrm{C}$. This high temperature range is in agreement with the only small degree of azide decomposition found during polymerization at $70{ }^{\circ} \mathrm{C}$. A massive mass loss above $350{ }^{\circ} \mathrm{C}$ indicated the decomposition of the polymer material under nitrogen atmosphere. This polymer decomposition temperature increased with initial content of azide groups (which, at temperatures above $350{ }^{\circ} \mathrm{C}$, were expected to have undergone crosslinking reactions). Interestingly and although the material was fully organic, a considerable percentage of mass that increased with azide content to over $25 \mathrm{wt} \%$, remained at $900{ }^{\circ} \mathrm{C}$ (Fig. S7 $\dagger$ ), suggesting considerable thermal stability conferred by the nitrene-based crosslinking.

\section{Controlled photo-crosslinking}

With a set of well-defined azide-functional microspheres in hand, their crosslinking through controlled UV irradiation was studied next. Upon irradiation, the azide group sheds a molecule of nitrogen and forms a highly reactive nitrene group which can undergo several addition and insertion reactions with nearby molecules forming covalent crosslinks between different polymer chains. Main expected reaction pathways for the crosslinking of azide-functional microspheres are shown in Scheme $3 .^{51}$ Notably, most of these reactions do not rely on dimerization (as do other common photo-crosslinkers) but offer universal attachment to nearby groups.

A UV lamp emitting a wavelength of $254 \mathrm{~nm}$, ideal for the formation of nitrenes from azides ${ }^{50}$ and designed for irradiation of liquid samples, was dipped directly into a stirred

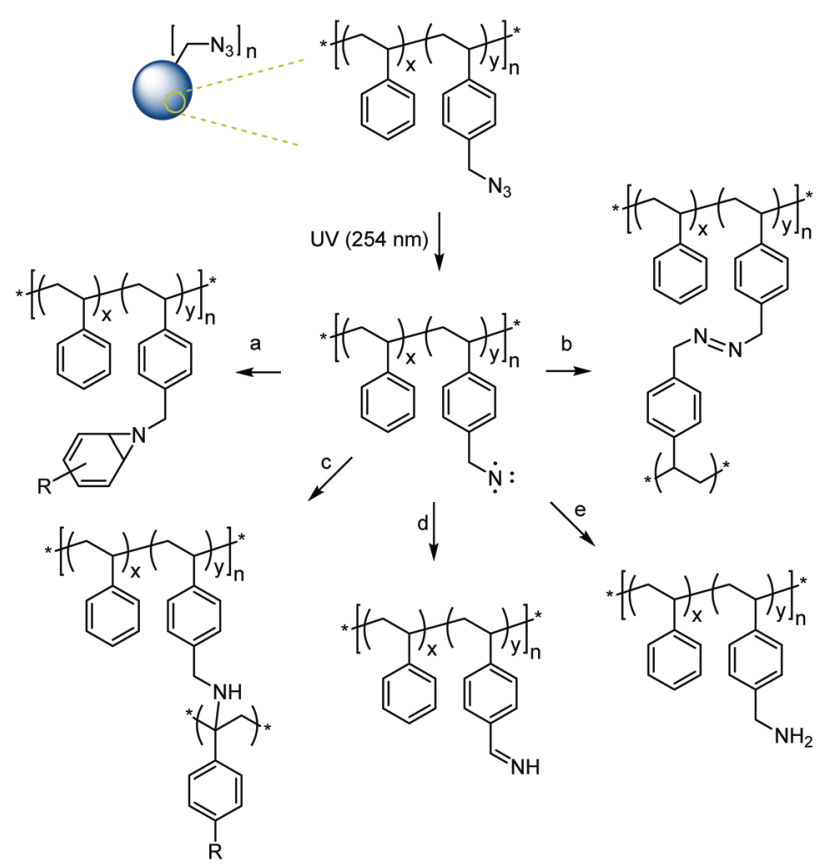

Scheme 3 Possible reactions of the singlet nitrene formed after azide irradiation: ${ }^{51}$ (a) addition to double bond; (b) nitrene-nitrene coupling; and (c) $\mathrm{C}-\mathrm{H}$ bond insertion (shown at benzylic position) leading to crosslinking and (d), (e) $\mathrm{H}$ abstractions not leading to crosslinking. suspension of particles in water which was kept at room temperature through a cooling tube dipped into the solution. Irradiation times were varied between $30 \mathrm{~s}$ and $1 \mathrm{~h}$ and the conversion based on the amount of residual azide functionality was determined by three methods: (i) FT-IR spectroscopy, through integration of the azide vibrational band at $2095 \mathrm{~cm}^{-1}$ and calibrating the absorbance of samples before irradiation to $100 \%$ (Fig. S6†); (ii) elemental analysis by determining the nitrogen content and taking into account the original nitrogen content before irradiation and the expected remainder of a third of this mass after quantitative reaction (Scheme 3); (iii) DSC by measuring the exothermic heat flow during the thermal degradation of residual azide groups using the above data (Fig. 7) for calibration. As a representative example, the azide conversion for the irradiation of sample $\mathrm{MS}_{50}$ as determined by IR, EA, and DSC is shown in Fig. 8. Under the reactions conditions, a conversion of $50 \%$ of azide groups was achieved in 3 minutes, a conversion of approx. 95\% was found after $20 \mathrm{~min}$ of irradiation, and essentially complete reaction was confirmed after $60 \mathrm{~min}$. Importantly, the data from all three analytical methods was in excellent agreement, confirming the reliability of each technique, which was also apparent through repeated experiments. Additionally, this agreement also indicated that the penetration depth of the UV light during irradiation was sufficient to perform reactions inside the microspheres.

For microsphere samples of varying azide loadings, the observed reaction rate increased with decreasing azide content, Fig. 9. For sample $\mathrm{MS}_{10}$, for example, (near-) complete reaction was found after 5 minutes of irradiation, while sample $\mathrm{MS}_{100}$ required $30 \mathrm{~min}$ to reach approx. 98\% conversion. Exponential fitting of data suggested first order kinetics for the azide decomposition of sample $\mathrm{MS}_{100}$, though notably,

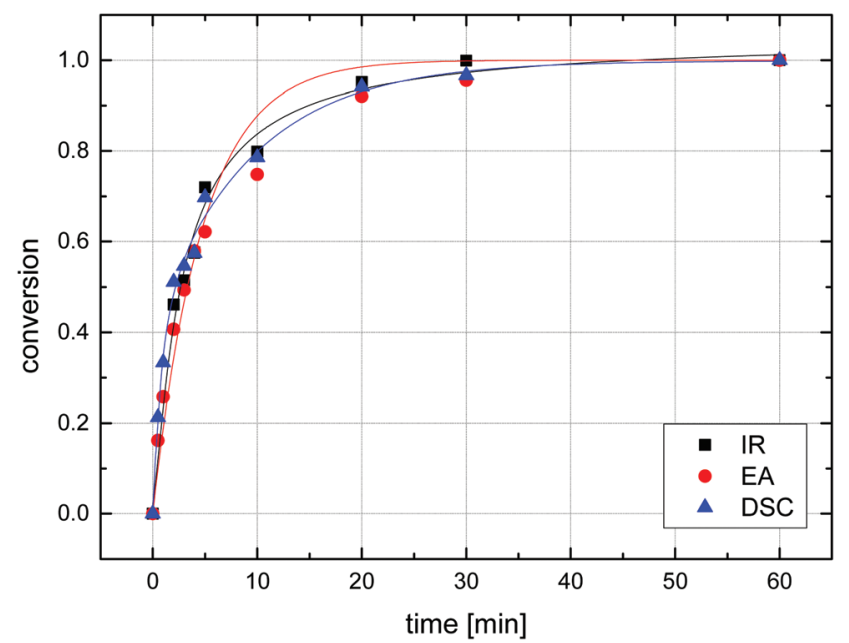

Fig. 8 Azide decomposition (conversion $=1-$ residual azide) vs. time during the irradiation of $\mathrm{P}(\mathrm{S}-\mathrm{co}-\mathrm{VBA})$ microsphere sample $\mathrm{MS}_{50}$ determined by FT-IR spectroscopy (squares), elemental analysis (circles), and differential scanning calorimetry (triangles). The curves are exponential fits of the data to guide the eye. 


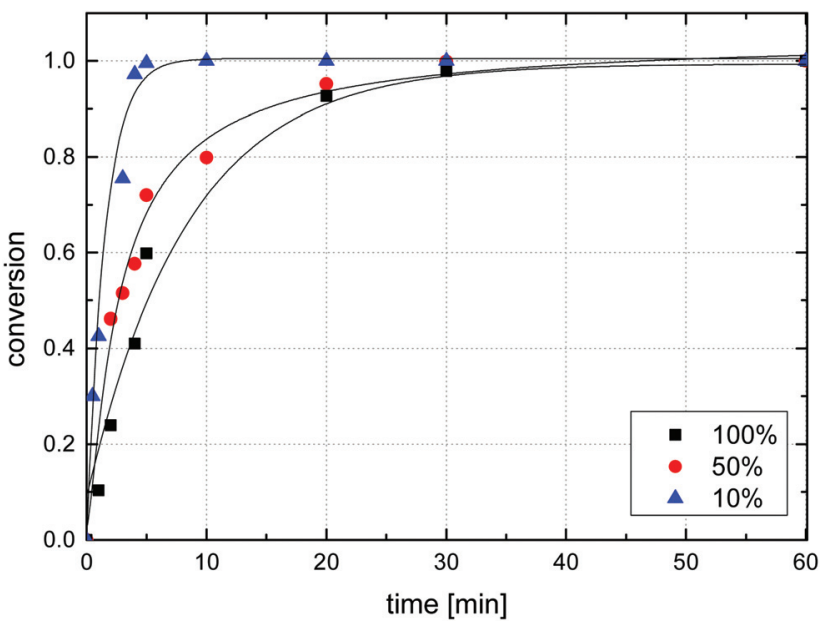

Fig. 9 Comparison of azide conversion during the irradiation of microsphere samples $\mathrm{MS}_{10}, \mathrm{MS}_{50}$, and $\mathrm{MS}_{100}$ determined by IR spectroscopy.

other samples deviated from first order kinetics with observed reaction rates generally lower than predicted by first-order theory, Fig. S8. $\uparrow$ The azide decomposition rate may be influenced by several factors including UV absorbance by the azide itself and the polystyrene matrix leading to lower flux of light inside particles, and a matrix absorptivity that depends on the crosslink density.

The local concentration of azides has also been shown to influence the preferred reaction pathway of the nitrene intermediates (Scheme 3) with crosslinking through nitrenenitrene coupling typically only found for high azide concentrations and pathways (a) and (c) (Scheme 3) being the most common crosslinking reactions at low or intermediate azide concentration..$^{51}$ Notably, some possible reaction pathways involving $\mathrm{H}$ abstraction from a $\mathrm{C}-\mathrm{H}$ bond without combination with the C-centered radical do not lead to crosslinking reac- tions. FT-IR measurements and ${ }^{13} \mathrm{C}$ solid state NMR spectroscopy were run on samples $\mathrm{MS}_{10}, \mathrm{MS}_{50}$, and $\mathrm{MS}_{100}$ before and after exhaustive crosslinking. Both measurements confirmed the disappearance of the azide group (disappearance of the $\mathrm{N}=\mathrm{N}=\mathrm{N}$ vibration at $2095 \mathrm{~cm}^{-1}$ in the IR spectrum and of the ${ }^{13} \mathrm{C}$ NMR resonance assigned to the $-\mathrm{CH}_{2} \mathrm{~N}_{3}$ group at 54.6 ppm, Fig. S9†). However, the results were inconclusive with regards to identifying preferred nitrene reaction pathways as shown in Scheme 3. While reaction pathway (a) (leading to crosslinking through $\mathrm{C}=\mathrm{C}$ addition) was expected to prevail, it was not possible to determine the exact crosslinking density from the azide conversion.

Notably, most published examples on azide-functional microspheres, films, or other architectures describe exhaustive irradiation and crosslinking. Significantly, the combination of a reproducible experimental setup, and use of either IR, EA, or DSC analysis described herein enabled the partial crosslinking of microspheres by sparing a defined and predetermined amount of azide functionality which can then be available for further modification. This protocol thus allows for tuning of the initial azide content (Table 1) and the proportion of azide functionality used for crosslinking. Taking advantage of this, microspheres with different extents of crosslinking and residual azide functionality were prepared and characterized by SEM imaging and swelling studies, Table 2 .

SEM analysis of irradiated microspheres showed that the spherical shapes, particle diameters, and particle size distributions were not affected by the photo-crosslinking. In fact, for most samples, SEM images showed no differences to the images recorded before crosslinking, see Fig. 10 and average particle diameters and dispersities given in Table 2. For two samples, $\mathrm{MS}_{50}$ and $\mathrm{MS}_{100}$, both with high azide loadings, however, exhaustive crosslinking for 60 minutes was found to result in particles being 'glued' together as observed by SEM, Fig. 11. Presumably, microspheres aggregated in their aqueous

Table 2 Overview of partially and fully crosslinked microsphere samples

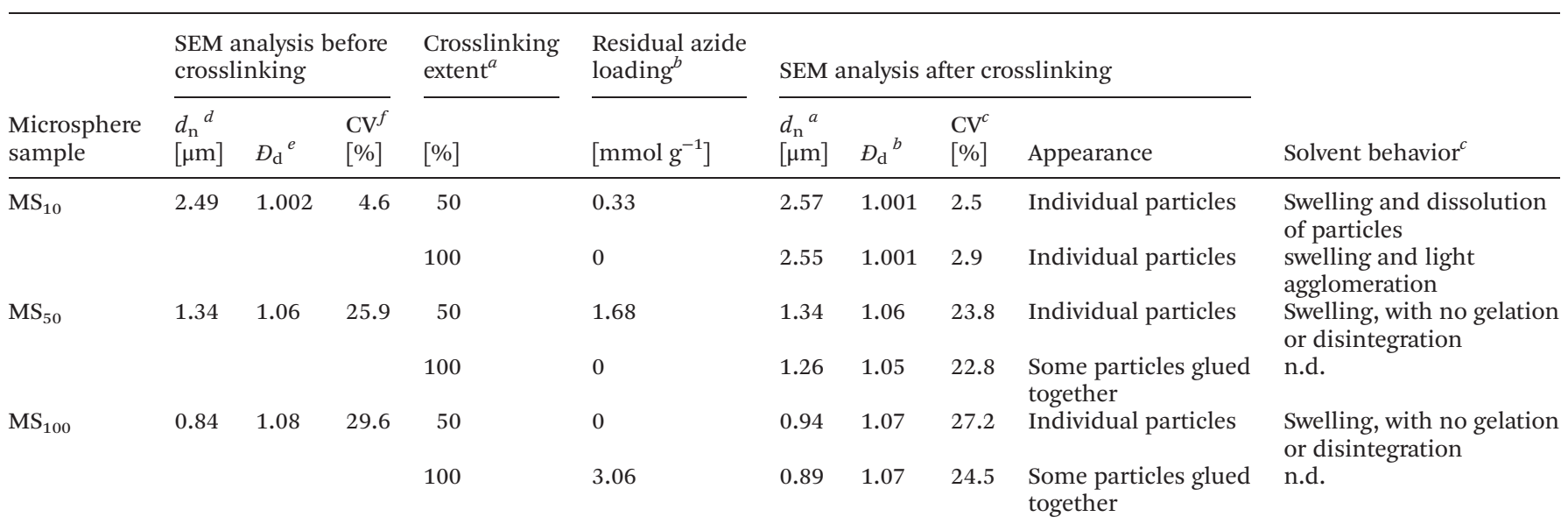

\footnotetext{
${ }^{a}$ Percentage of azide functionality used during irradiation determined through FT-IR analysis of the residual azide band at $2095 \mathrm{~cm}^{-1}$.

${ }^{b}$ Estimated from initial azide loading, azide decomposition and average molar mass. ${ }^{c}$ Crosslinked microspheres were kept in THF for 24 h.

${ }^{d}$ Number-average particle diameter. ${ }^{e}$ Dispersity of the particle diameter. ${ }^{f}$ Coefficient of variation of the particle diameter.
} 

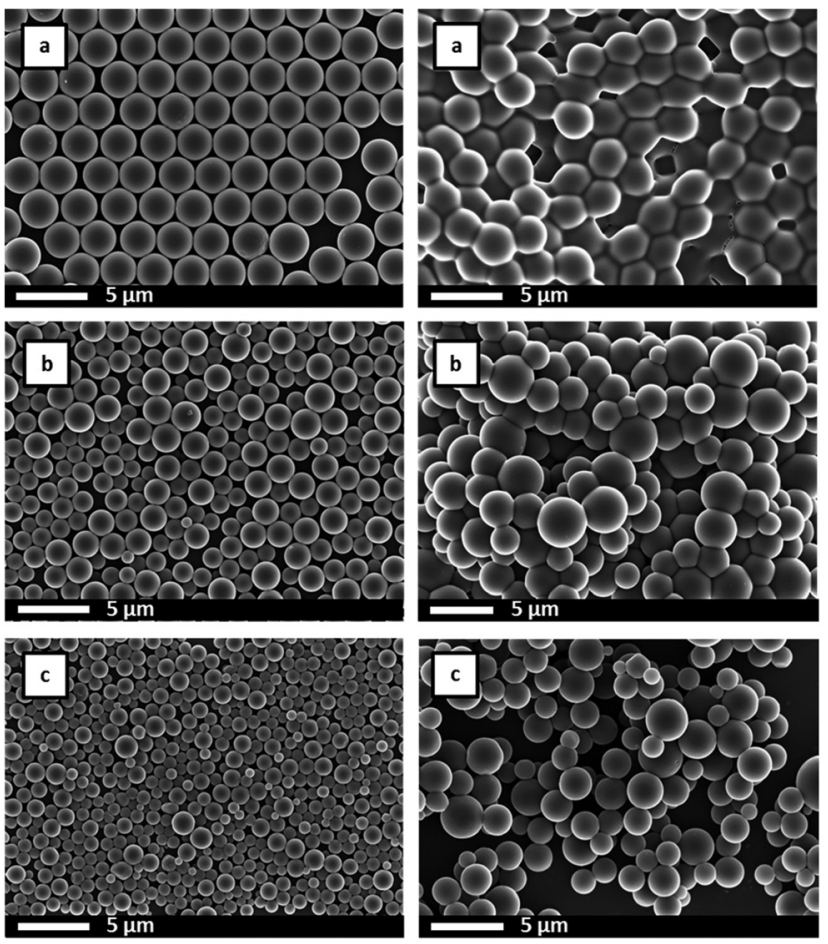

Fig. 10 SEM images of photo-crosslinked microspheres after irradiation (left images) and after stirring in THF for $24 \mathrm{~h}$ showing swelling (right images) of samples (a) $\mathrm{MS}_{10}$ after $100 \%$ azide conversion; (b) $\mathrm{MS}_{50}$ with $50 \%$ of azide functionality used for crosslinking and (c) $\mathrm{MS}_{100}$ with $50 \%$ of azide functionality used for crosslinking. Photo-crosslinking was not found to affect the size dispersity of microspheres while swelling of particles with a low degree of crosslinking was observed in THF.

suspension during the irradiation and nitrene reactions formed covalent links between particles.

Successful crosslinking was demonstrated by adding irradiated microspheres into THF (a good solvent for the polystyrene-based copolymers that dissolves non-crosslinked particles). The sample with the lowest crosslinking density, $\mathrm{MS}_{10}$ after using $100 \%$ of azide functionality for crosslinking swelled considerably with particles sticking together as seen by SEM analysis, Fig. 10 (top right). For all other samples, particles did not disintegrate in THF indicating sufficient crosslinking to prevent dissolution. These observations suggested that more than $10 \mathrm{wt} \%$ VBA feed were necessary to
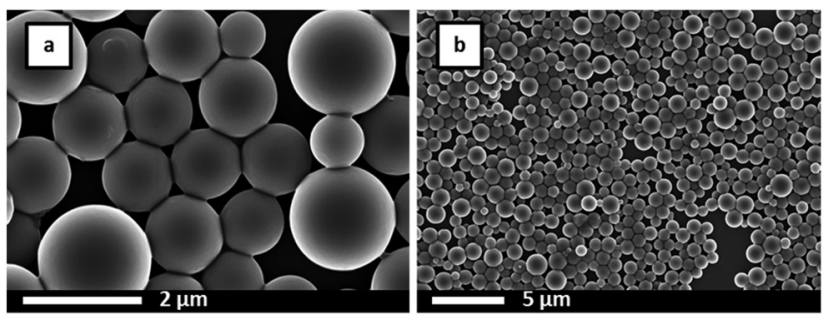

Fig. 11 SEM images of microspheres (a) $M S_{50}$ and (b) $M S_{100}$ each after UV irradiation for $60 \mathrm{~min}$ leading to some extent of particle agglomeration. ensure adequate crosslinking and that for samples containing $50 \mathrm{wt} \%$ or more of VBA, irradiation times should be kept below $60 \mathrm{~min}$ under these reaction conditions to prevent particle agglomeration.

\section{Click modification of partially crosslinked microspheres}

As mentioned above, the chemical modification of microspheres is crucial for many applications and the Cu-catalyzed azide-alkyne cycloaddition (CuAAC) reaction has been documented to be amenable to the modification of azide (and alkyne $)^{43}$-functional microspheres. Herein, after demonstrating the preparation of microspheres with a tunable degree of crosslinking and a tunable amount of residual azide functionality through partial photo-crosslinking, the chemical accessibility of the residual azide groups was demonstrated through CuAAC-modification with an alkyne-functional fluorescent dye, Rhodamine B propargyl ester, Scheme 1. This alkyne was chosen because of the obvious visual confirmation of successful modification, the importance of fluorescent microspheres as probes for optical measurements, but also because, as a larger, sterically hindered alkyne, it represents a suitable way of probing the accessibility of the azide groups with challenging alkyne reagents. As substrate, a sample of $\mathrm{MS}_{50}$, photocrosslinked for $3 \mathrm{~min}$ and with $48 \%$ of residual azide functionality (as determined by IR-spectroscopy), was chosen. Rhodamine B propargyl ester was provided in 3-fold excess to azide groups and $\mathrm{CuBr}, N, N, N^{\prime}, N^{\prime}$-tetramethylethylendiamine, and triethylamine were used as the catalytic system in DMF as solvent at room temperature. ${ }^{35,43}$ After intensive washing of the dye-modified microspheres until washings were colorless, the product was obtained as a deeply purple powder, Fig. 12. IR spectroscopy, Fig. 13, indicated a remainder of approx. 9\% of the initial azide content. As CuAAC represents the only viable reaction that diminishes the azide concentration, this data suggested that after $52 \%$ of azide groups had been used for crosslinking, another $39 \%$ of initial azide groups were modified through CuAAC, or that $81 \%$ of the azide groups spared during photo-crosslinking were successfully conjugated

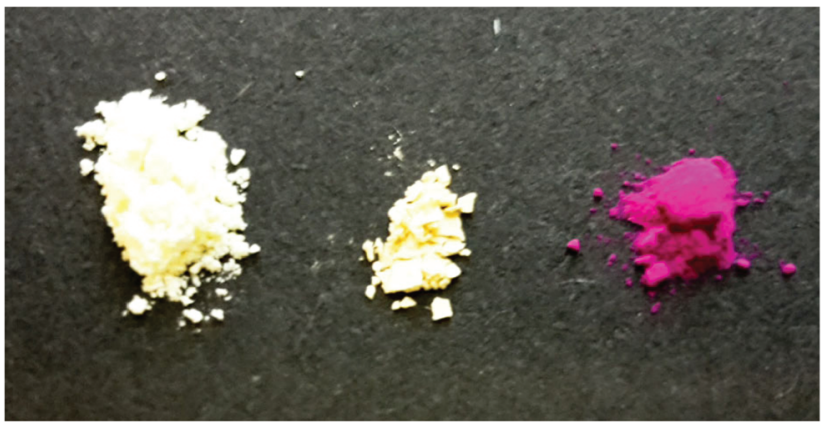

Fig. 12 Photograph of microspheres $M_{50}$ as synthesized, featuring azide-functionality and presenting as an off-white powder (left); after partial photo-crosslinking, presenting as slightly more yellow powder (middle); and after modification of the residual azide groups with Rhodamine B propargyl ester showing bright purple color (right). 


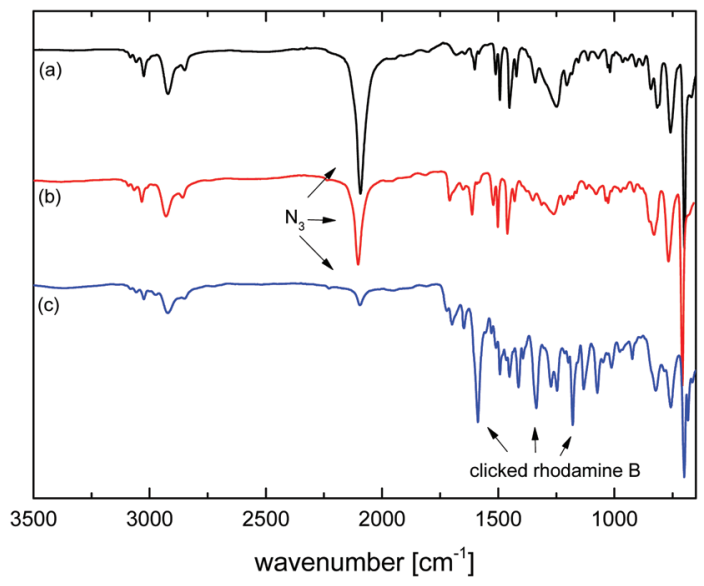

Fig. 13 FT-IR spectra of microspheres $\mathrm{MS}_{50}$ (a) as synthesized, (b) after partial photo-crosslinking for 3 min sparing $48 \%$ of azide functionality, and (c) after CuAAC modification with Rhodamine B propargyl ester after which $9 \%$ of initial azide functionality remained. The $\mathrm{N}=\mathrm{N}=\mathrm{N}$ asymmetric stretch of the azide group and characteristic vibrations of the dye are marked.

to the fluorescent dye-a fairly high reaction efficiency when comparing to the literature and considering the bulky nature of the dye alkyne. ${ }^{35,43}$ The IR spectrum run on the dyemodified microsphere sample additionally showed characteristic vibrations of the Rhodamine dye, qualitatively confirming its presence in the modified particles.

\section{Conclusions}

This study exploited several key characteristics of the azide functionality for the preparation of crosslinked and functionalized polystyrene-based microspheres: (i) while the benzyl chloride functional group was found to undergo nucleophilic substitution with the alcoholic solvent during polymerization, the benzyl azide functional group was found to be stable under the same reaction conditions. This allowed for a first description of dispersion (co-)polymerizations based on vinylbenzyl azide to give well-defined microspheres with tunable azide loadings. (ii) Upon irradiation at $254 \mathrm{~nm}$, the azide group forms a highly reactive nitrene intermediate that can undergo a combination of reactions with nearby $\mathrm{C}-\mathrm{H}$ and $\mathrm{C}=\mathrm{C}$ bonds. This was exploited to crosslink the microspheres while preserving their sizes and size distributions. (iii) As documented in the literature for the modification of microspheres, the azide group is amenable to highly efficient copper-catalyzed cycloaddition reactions with alkynes, which was used herein to conjugate residual azide functionality to a fluorescent dye. The pivotal point of this work is that the photo-crosslinking can be done under controlled conditions that allow for the conversion of a predetermined amount of azide functionality and that this conversion can be easily monitored by FT-IR spectroscopy, elemental analysis, and differential scanning calorimetry. As a result, the degree of crosslinking and the amount of unreacted azide functionality can be tuned and the azide group, as a single functionality, can be exploited for both photo-crosslinking and click-modification. Given the inherent difficulties in producing crosslinked and functional microspheres associated with many common synthetic strategies, the presented methodology offers a simple, robust, and versatile avenue toward well-defined microsphere materials that can be customized depending on application requirements.

\section{Acknowledgements}

The authors thank Renate Walter (Zoological Institute, University of Hamburg) for SEM measurements. Dr Young Joo Lee at the NMR center of the Chemistry Department, University of Hamburg is acknowledged for solid state NMR measurements. M. A., W. P., and H.-U. M. acknowledge the German Federal Ministry of Education and Research (BMBF) for financial support (13N11312).

\section{References}

1 J. Wang, P. A. G. Cormack, D. C. Sherrington and E. Khoshdel, Monodisperse, Molecularly Imprinted Polymer Microspheres Prepared by Precipitation Polymerization for Affinity Separation Applications, Angew. Chem., Int. Ed., 2003, 42(43), 5336-5338.

2 M. R. Buchmeiser, New synthetic ways for the preparation of high-performance liquid chromatography supports, J. Chromatogr., A, 2001, 918(2), 233-266.

3 E. Unsal, S. T. Camli, S. Senel and A. Tuncel, Chromatographic performance of monodisperse-macroporous particles produced by "modified seeded polymerization." I: Effect of monomer/seed latex ratio, J. Appl. Polym. Sci., 2004, 92(1), 607-618.

4 K. Saralidze, L. H. Koole and M. L. W. Knetsch, Polymeric Microspheres for Medical Applications, Materials, 2010, 3(6), 3537-3564.

5 A. Yethiraj, Tunable colloids: control of colloidal phase transitions with tunable interactions, Soft Matter, 2007, 3(9), 1099-1115.

6 B. Peng, E. van der Wee, A. Imhof and A. van Blaaderen, Synthesis of Monodisperse, Highly Cross-Linked, Fluorescent PMMA Particles by Dispersion Polymerization, Langmuir, 2012, 28(17), 6776-6785.

7 D. Zanaga, F. Bleichrodt, T. Altantzis, N. Winckelmans, W. J. Palenstijn, J. Sijbers, B. de Nijs, M. A. van Huis, A. Sanchez-Iglesias, L. M. Liz-Marzan, A. van Blaaderen, K. Joost Batenburg, S. Bals and G. Van Tendeloo, Quantitative 3D analysis of huge nanoparticle assemblies, Nanoscale, 2016, 8(1), 292-299.

8 H. Kawaguchi, Functional polymer microspheres, Prog. Polym. Sci., 2000, 25(8), 1171-1210. 
9 N. Fontanals, P. Manesiotis, D. C. Sherrington and P. A. G. Cormack, Synthesis of Spherical Ultra-HighSurface-Area Monodisperse Amphipathic Polymer Sponges in the Low-Micrometer Size Range, Adv. Mater., 2008, 20(7), 1298-1302.

10 J. Ugelstad, L. Soderberg, A. Berge and J. Bergstrom, Monodisperse polymer particles - a step forward for chromatography, Nature, 1983, 303(5912), 95-96.

11 R. P. A. Dullens, E. M. Claesson and W. K. Kegel, Preparation and Properties of Cross-Linked Fluorescent Poly (methyl methacrylate) Latex Colloids, Langmuir, 2004, 20(3), 658-664.

12 A. Tuncel, A. Denizli, M. Abdelaziz, H. Ayhan and E. Piskin, Biologically Modified Polymeric Biomaterial SurfacesAlbumin adsorption on to large-size monodisperse polystyrene latices having functional groups on their surfaces, Clin. Mater., 1992, 11(1), 139-144.

13 R. Arshady, Suspension, emulsion, and dispersion polymerization: A methodological survey, Colloid Polym. Sci., 1992, 270(8), 717-732.

14 C. M. Cheng, F. J. Micale, J. W. Vanderhoff and M. S. El-Aasser, Synthesis and characterization of monodisperse porous polymer particles, J. Polym. Sci., Part A: Polym. Chem., 1992, 30(2), 235-244.

15 M. T. Gokmen and F. E. Du Prez, Porous polymer particles -A comprehensive guide to synthesis, characterization, functionalization and applications, Prog. Polym. Sci., 2012, 37(3), 365-405.

16 M. J. Derry, L. A. Fielding and S. P. Armes, Polymerizationinduced self-assembly of block copolymer nanoparticles via RAFT non-aqueous dispersion polymerization, Prog. Polym. Sci., 2016, 52, 1-18.

17 J. Rieger, Guidelines for the Synthesis of Block Copolymer Particles of Various Morphologies by RAFT Dispersion Polymerization, Macromol. Rapid Commun., 2015, 36(16), 1458-1471.

18 J.-T. Sun, C.-Y. Hong and C.-Y. Pan, Recent advances in RAFT dispersion polymerization for preparation of block copolymer aggregates, Polym. Chem., 2013, 4(4), 873-881.

19 J. Ugelstad, A. Berge, T. Ellingsen, O. Aune, L. Kilaas, T. N. Nilsen, R. Schmid, P. Stenstad, S. Funderud, G. Kvalheim, K. Nustad, T. Lea, F. Vartdal and H. Danielsen, Monosized magnetic particles and their use in selective cell separation, Makromolekulare Chemie, Macromol. Symp., 1988, 17(1), 177-211.

20 J. Ugelstad, K. H. Kaggerud, F. K. Hansen and A. Berge, Absorption of low molecular weight compounds in aqueous dispersions of polymer-oligomer particles, 2. A two step swelling process of polymer particles giving an enormous increase in absorption capacity, Die Makromol. Chem., 1979, 180(3), 737-744.

21 J. Ugelstad, H. R. Mfutakamba, P. C. Mørk, T. Ellingsen, A. Berge, R. Schmid, L. Holm, A. Jørgedal, F. K. Hansen and K. Nustad, Preparation and application of monodisperse polymer particles, J. Polym. Sci., Polym. Symp., 1985, 72(1), 225-240.
22 J. Ugelstad, P. C. Mórk, K. H. Kaggerud, T. Ellingsen and A. Berge, Swelling of oligomer-polymer particles. New methods of preparation, Adv. Colloid Interface Sci., 1980, 13(1-2), 101-140.

23 H. Bamnolker and S. Margel, Dispersion polymerization of styrene in polar solvents: Effect of reaction parameters on microsphere surface composition and surface properties, size and size distribution, and molecular weight, J. Polym. Sci., Part A: Polym. Chem., 1996, 34(10), 1857-1871.

24 C.-W. Chen and C.-Y. Chen, Preparation of monodisperse polystyrene microspheres: effect of reaction parameters on particle formation, and optical performances of its diffusive agent application, Colloid Polym. Sci., 2009, 287(12), 1377-1389.

25 S. T. Ha, O. O. Park and S. H. Im, Size control of highly monodisperse polystyrene particles by modified dispersion polymerization, Macromol. Res., 2010, 18(10), 935-943.

26 S. Kawaguchi and K. Ito, Dispersion Polymerization, Adv. Polym. Sci., 2005, 175, 299-328.

27 S. M. Klein, V. N. Manoharan, D. J. Pine and F. F. Lange, Preparation of monodisperse PMMA microspheres in nonpolar solvents by dispersion polymerization with a macromonomeric stabilizer, Colloid Polym. Sci., 2003, 282(1), 7-13.

28 K. P. Lok and C. K. Ober, Particle size control in dispersion polymerization of polystyrene, Can. J. Chem., 1985, 63(1), 209-216.

29 H. Uyama and S. Kobayashi, Dispersion polymerization of styrene in aqueous alcohol solution: Effects of reaction parameters on the polymer particle formation, Polym. Int., 1994, 34(3), 339-344.

30 J. Choi, S. Y. Kwak, S. Kang, S. S. Lee, M. Park, S. Lim, J. Kim, C. R. Choe and S. I. Hong, Synthesis of highly crosslinked monodisperse polymer particles: Effect of reaction parameters on the size and size distribution, J. Polym. Sci., Part A: Polym. Chem., 2002, 40(23), 4368-4377.

31 J.-S. Song, F. Tronc and M. A. Winnik, Two-Stage Dispersion Polymerization toward Monodisperse, Controlled Micrometer-Sized Copolymer Particles, J. Am. Chem. Soc., 2004, 126(21), 6562-6563.

32 J.-S. Song and M. A. Winnik, Cross-Linked, Monodisperse, Micron-Sized Polystyrene Particles by Two-Stage Dispersion Polymerization, Macromolecules, 2005, 38(20), 8300-8307.

33 C. M. Tseng, Y. Y. Lu, M. S. El-Aasser and J. W. Vanderhoff, Uniform polymer particles by dispersion polymerization in alcohol, J. Polym. Sci., Part A: Polym. Chem., 1986, 24(11), 2995-3007.

34 J. Tan, X. Rao, X. Wu, H. Deng, J. Yang and Z. Zeng, Photoinitiated RAFT Dispersion Polymerization: A Straightforward Approach toward Highly Monodisperse Functional Microspheres, Macromolecules, 2012, 45(21), 8790-8795.

35 M. Albuszis, P. J. Roth, F. Exnowitz, D. L. Wong, W. Pauer and H.-U. Moritz, Synthesis and in-depth characterization of reactive, uniform, crosslinked microparticles based on free radical copolymerization of 4-vinylbenzyl azide, Polym. Chem., 2016, 7(5), 1168-1180. 
36 T. Bahar and A. Tuncel, Monodisperse poly(p-chloromethylstyrene) microbeads by dispersion polymerization, Polym. Eng. Sci., 1999, 39(10), 1849-1855.

37 S. Margel, E. Nov and I. Fisher, Polychloromethylstyrene microspheres: Synthesis and characterization, J. Polym. Sci., Part A: Polym. Chem., 1991, 29(3), 347-355.

38 J.-P. Montheard, M. Chatzopoulos and M. Camps, Chemical Transformations of Chloromethylated Polystyrene, J. Macromol. Sci., Part C: Polym. Rev., 1988, 28(3-4), 503592.

39 I. Dumistracel, G. Ponchel, G. Danila, D. Duchene and A. Carpov, Poly(vinylbenzyl chloride) microsphere synthesis and their chemical modifications, J. Microencapsulation, 2000, 17(1), 45-55.

40 B. Elmas, S. T. Camli, M. Tuncel, S. Senel and A. Tuncel, DNA-responsive uniform latex particles based on p-chloromethylstyrene, J. Biomater. Sci., Polym. Ed., 2012, 12(3), 283-296.

41 Ç. Kip, B. Maraş, O. Evirgen and A. Tuncel, A new type of monodisperse porous, hydrophilic microspheres with reactive chloroalkyl functionality: synthesis and derivatization properties, Colloid Polym. Sci., 2013, 292(1), 219-228.

42 A. Zillessen and E. Bartsch, Synthesis of Photo-Cross-Linkable Microgel Colloids for Cluster Formation Studies, Langmuir, 2010, 26(1), 89-96.

43 M. Albuszis, P. J. Roth, W. Pauer and H.-U. Moritz, Macroporous uniform azide- and alkyne-functional polymer microspheres with tuneable surface area: synthesis, indepth characterization and click-modification, Polym. Chem., 2014, 5(19), 5689-5699.

44 A. Bayraktar, B. Saracoglu, C. Golgelioglu and A. Tuncel, Click-chemistry for surface modification of monodispersemacroporous particles, J. Colloid Interface Sci., 2012, 365(1), 63-71.

45 D. R. Breed, R. Thibault, F. Xie, Q. Wang, C. J. Hawker and D. J. Pine, Functionalization of polymer microspheres using click chemistry, Langmuir, 2009, 25(8), 4370-4376.

46 K. Ouadahi, E. Allard, B. Oberleitner and C. Larpent, Synthesis of azide-functionalized nanoparticles by microemulsion polymerization and surface modification by click chemistry in aqueous medium, J. Polym. Sci., Part A: Polym. Chem., 2012, 50(2), 314-328.
47 V. V. Rostovtsev, L. G. Green, V. V. Fokin and K. B. Sharpless, A Stepwise Huisgen Cycloaddition Process: Copper(I)-Catalyzed Regioselective "Ligation" of Azides and Terminal Alkynes, Angew. Chem., Int. Ed., 2002, 41(14), 2596-2599.

48 V. Rodionov, H. Gao, S. Scroggins, D. A. Unruh, A.-J. Avestro and J. M. J. Fréchet, Easy Access to a Family of Polymer Catalysts from Modular Star Polymers, J. Am. Chem. Soc., 2010, 132(8), 2570-2572.

49 M. K. Klein, A. Zumbusch and P. Pfleiderer, Photo-crosslinkable, deformable PMMA colloids, J. Mater. Chem. C, 2013, 1(43), 7228-7236.

50 L. Xu, J. Farrell, R. G. Karunakaran, A. Honglawan and S. Yang, Synthesis of dual-functional copolymer with orthogonally photosensitive groups, J. Polym. Sci., Part A: Polym. Chem., 2013, 51(5), 1215-1222.

51 S. Al Akhrass, R.-V. Ostaci, Y. Grohens, E. Drockenmuller and G. Reiter, Influence of Progressive Cross-Linking on Dewetting of Polystyrene Thin Films, Langmuir, 2008, 24(5), 1884-1890.

52 H. Morita, S. Mori, N. Uchino and S. Yokoyama, Magnetic Field and Polymer Matrix Effects on Photocrosslinking Reaction in Solid Polymer Matrices, in Zeitschrift für Physikalische Chemie, 1993, vol. 182, p. 209.

53 Q. Zhang, Y. Han, W. Wang, T. Song and J. Chang, A theoretical and experimental investigation of the size distribution of polystyrene microspheres by seeded polymerization, J. Colloid Interface Sci., 2010, 342(1), 62-67.

54 D. Horák and P. Shapoval, Reactive poly(glycidyl methacrylate) microspheres prepared by dispersion polymerization, J. Polym. Sci., Part A: Polym. Chem., 2000, 38(21), 3855-3863.

55 V. Ladmiral, T. M. Legge, Y. Zhao and S. Perrier, "Click" Chemistry and Radical Polymerization: Potential Loss of Orthogonality, Macromolecules, 2008, 41(18), 6728-6732.

56 Y. Li, J. Yang and B. C. Benicewicz, Well-controlled polymerization of 2-azidoethyl methacrylate at near room temperature and click functionalization, J. Polym. Sci., Part A: Polym. Chem., 2007, 45(18), 4300-4308.

57 R. J. Andrews and E. A. Grulke, Glass Transition Temperatures of Polymers, in Polymer Handbook ed. J. Brandrup, E. H. Immergut and E. A. Grulke, John Wiley \& Sohns, fourth edition, 1999. 